\title{
SPARSE SPECTRAL ANALYSIS OF ATRIAL FIBRILLATION ELECTROGRAMS
}

\author{
Sandra Monzón, Tom Trigano , David Luengo and Antonio Artés-Rodríguez
}

\begin{abstract}
Atrial fibrillation (AF) is a common heart disorder. One of the most prominent hypothesis about its initiation and maintenance considers multiple uncoordinated activation foci inside the atrium. However, the implicit assumption behind all the signal processing techniques used for $\mathrm{AF}$, such as dominant frequency and organization analysis, is the existence of a single regular component in the observed signals. In this paper we take into account the existence of multiple foci, performing a spectral analysis to detect their number and frequencies. In order to obtain a cleaner signal on which the spectral analysis can be performed, we introduce sparsity-aware learning techniques to infer the spike trains corresponding to the activations. The good performance of the proposed algorithm is demonstrated both on synthetic and real data.
\end{abstract}

\section{INTRODUCTION}

The clinical term atrial fibrillation (AF) refers to a family of common heart disorders characterized by fast and uncoordinated activations in the atrium. Several theories about the physiological causes underlying AF initiation and maintenance have been formulated over the last 50 years [1]. One of the most prominent hypothesis considers multiple activation foci placed at different locations inside the atrium. These fast and asynchronous activations cause a disordered global electrical activity that contributes to AF maintenance. In contrast, during normal heart operation conditions (sinus rhythm) we observe a single activation focus, placed at the sinus node, acting as a pacemaker for the whole heart and leading to a regular global electrical activity.

In order to understand the pathophysiology of AF, dominant frequency analysis (DFA) has been traditionally used to analyze the data collected from electrocardiograms (ECGs) or electrograms (EGMs). DFA is useful for identifying the areas corresponding to the highest activation frequencies that may be the drivers maintaining $\mathrm{AF}$, and therefore the targets of ablation therapy for AF termination [2]. However, DFA provides very limited information about the signal's structure, since it is based on the implicit assumption that the underlying signal consists of a single quasi-periodic component plus an irregular component [3]. Hence, the only spectral parameter required is the dominant frequency (DF), which tries to characterize the periodicity of the signal, but is very sensitive to distortions and often provides misleading information [4].

More recently, organization analysis techniques have been introduced, and additional parameters, such as the regularity index (RI) and the organization index (OI), have been used to describe the signals [5]. Many other linear and non-linear measures have been proposed for the characterization of $\mathrm{AF}$ $[6,7]$ : the cross-correlation index, the non-linear association measure, the fractionation index, etc. However, all of them are still based on the same implicit assumption: the observed signals can be modelled by a single regular component plus distortion and noise.

In this paper we make two main contributions. First of all, we introduce a more realistic mathematical model that takes into account the multiple activation foci, and use it to perform spectral analysis, detecting the number of foci and their frequencies. And secondly, recognizing the sparse nature of the recorded signals, we apply a sparsity-aware learning technique (LASSO) to obtain an activation sequence on which the spectral analysis is performed. The resulting algorithm consists of three steps: inferring the spike trains associated to the activation times using a sparsity-aware learning technique based on LASSO [8]; sparse spectral analysis of that activation sequence, using an iterative deflation approach to detect the number of foci and their frequencies; and post-processing in order to eliminate harmonics and subharmonics.

The paper is structured as follows. In Section 2 we provide a brief overview of the traditional DFA approach. Then, Section 3 describes our approach: sparse spectral analysis (SSA). We start showing the mathematical model considered, followed by a detailed description of the three steps of the algorithm. Simulation results are presented in Section 4, both for synthetic data and for real EGMs, obtained both under sinus rhythm conditions and atrial fibrillation. 


\section{DOMINANT FREQUENCY ANALYSIS}

\subsection{Mathematical Model}

DFA assumes implicitly that the observed signals are composed of a single regular component (i.e. a quasi-periodic signal) plus an irregular component including the remaining noise and distortion. Hence, from a mathematical point of view, the $q$-th output (EGM), $1 \leq q \leq Q$ with $Q$ denoting the number of outputs, can be modelled as [5]

$$
y_{q}(t)=\sum_{k=-\infty}^{\infty} \phi_{q}\left(t-k \widetilde{T}_{q}-\tilde{\tau}_{q}\right)+w_{q}(t)
$$

where $\phi_{q}(t)$ indicates the average shape of the regular component of the signal, with $\widetilde{T}_{q}$ denoting its period and $\tilde{\tau}_{q}$ the delay for $k=0$, and $w_{q}(t)$ is used to represent the irregular components. The goal of DFA is characterizing that quasiperiodic signal through its average period, $\widetilde{T}_{q}$, or equivalently its average frequency, $\tilde{f}_{q}=1 / \tilde{T}_{q}$, which is the so called dominant frequency (DF). Occasionally other parameters, such as the organization or the regularity indexes, are also obtained to determine whether the estimated DF is reliable or not $[3,5]$.

\subsection{Spectral Analysis}

The DF is usually obtained separately for each channel using standard spectral analysis techniques. The typical signal processing approach includes five steps for each EGM [5]:

1. Band-Pass filtering from $30 \mathrm{~Hz}$ to $400 \mathrm{~Hz}$.

2. Rectification of the resulting signal, recovering near direct current (DC) spectral components.

\section{Low-Pass filtering with a cut-off frequency of $15 \mathrm{~Hz}$.}

4. Computation of the spectrum using a localized Fast Fourier Transform (FFT) with a Hanning window of $\Lambda=4 \mathrm{~s}$ duration, resulting in a resolution $f_{\Lambda}=1 / \Lambda=$ $0.25 \mathrm{~Hz}$ in the frequency domain.

5. Search for the peak with the maximum amplitude in the frequency domain. The frequency associated to this peak is the dominant frequency of the $q$-th EGM, $\tilde{f}_{q}$.

Several segments can be averaged in order to improve the estimation of the dominant frequency. However, the ability of the DF to reflect the average atrial activation rate depends on the accuracy of (1) in representing the true observed signal. Unfortunately, several characteristics of atrial activation, such as the complexity of the electrogram morphology, can alter the power spectrum. In these cases, the DF, $\tilde{f}_{q}$, is often more related to the complexity of the signal than to the atrial activation rate, thus providing misleading information [4].

\section{SPARSE SPECTRAL ANALYSIS}

\subsection{Mathematical Model}

Our basic assumption is that the recorded EGMs are composed of the sum of several periodic or quasi-periodic signals plus distortion and noise. Each of these observed periodic signals are the result of a set of sparse activation foci (spike trains) that propagate through the atrium and reach the sensors. Our goal is detecting the number of activation foci, as well as their frequencies.

From a mathematical point of view, let us consider again a model with $Q$ correlated outputs, $y_{q}(t)$, obtained from a set of bipolar electrodes. These observations are generated by $R$ activation foci (latent signals) propagating inside the atrium, plus noise and interference. Hence, we model the output of the $q$-th channel $(1 \leq q \leq Q)$ as

$$
y_{q}(t)=\sum_{r=1}^{R} p_{r}(t) * h_{r, q}(t)+w_{q}(t)
$$

where $p_{r}(t)(1 \leq r \leq R)$ denotes the $r$-th foci, $w_{q}(t)$ models all the elements in the $q$-th output that cannot be explained by the model (i.e. noise, interferences and distortion), $h_{r, q}(t)$ is the impulse response of the channel between the $r$-th foci and the $q$-th output EGM and $*$ denotes the standard linear convolution operator. ${ }^{1}$ Since we are not interested in recovering the precise shape of the activations, but only in their number and frequencies, we model them as periodic spike trains,

$$
p_{r}(t)=\sum_{k=-\infty}^{\infty} \delta\left(t-k T_{r}-\tau_{r}\right),
$$

with $\delta(t)$ denoting Dirac's delta function, $T_{r}$ the period of the $r$-th spike train and $\tau_{r}$ its shift w.r.t. the origin. ${ }^{2}$ Finally, substituting (3) into (2), the $q$-th output becomes

$$
y_{q}(t)=\sum_{r=1}^{R} \sum_{k=-\infty}^{\infty} h_{r, q}\left(t-k T_{r}-\tau_{r}\right)+w_{q}(t),
$$

which is an extension of the model assumed in DFA, given by (1). In the sequel we focus on inferring the global spike train (i.e. the spike train resulting from the sum of the $R$ foci), and using it to estimate $R$ and $f_{r}=1 / T_{r}$ for $r=1, \ldots, R$.

\subsection{Inference of the Sparse Activations}

The first step of the algorithm is a pre-processing stage that extracts the activations by exploiting two fundamental characteristics of EGM signals. Firstly, due to physiological causes

\footnotetext{
${ }^{1}$ Note that $h_{r, q}(t)$ includes the response of the sensor and is slowly timevarying. However, since the spectral analysis is performed using short time windows, we can consider the channel to be time-invariant in practice.

${ }^{2}$ Note that $T_{r}$ in (3) refers to the period of the $r$-th latent foci, whereas $\widetilde{T}_{q}$ in (1) denoted the average period of the regular component of the $q$-th output.
} 
(i.e. the existence of a refractory period [1]) the pulses cannot overlap. Secondly, the relevant information (activation times) is very localized. These two remarks lead us to consider an estimation approach based on sparse regression techniques. Thus, we propose applying a variation of the algorithm introduced in [9], which is described next.

Let us denote the vector with the $N+1$ samples from the $q$-th EGM by $\mathbf{y}_{q}=\left[y_{q}[0], y_{q}[1], \ldots, y_{q}[N]\right]^{\top}$, with $y_{q}[n]=$ $y_{q}\left(n T_{s}\right)$ obtained by sampling $y_{q}(t)$ uniformly with a sampling frequency $f_{s}=1 / T_{s}$. We define the vector containing the discrete-time differentiation of the $q$-th output, $\mathbf{z}_{q}=$ $\left[z_{q}[1], z_{q}[2], \ldots, z_{q}[N]\right]^{\top}$ with $z_{q}[n]=y_{q}[n]-y_{q}[n-1]$ for $1 \leq n \leq N$. The differentiation step is used to remove the baseline of the signal, so that the regression procedure can be performed on the mixture of noisy spikes. In the sequel we always work with $z_{q}[n]$ and, since we are not interested in the precise shape of the activations and the number of latent foci is still unknown, we approximate $z_{q}[n]$ by a mixture of shifted smooth generic curves:

$$
z_{q}[n]=\sum_{m=1}^{R} \sum_{k=-\infty}^{\infty} \beta_{m, q}[k] G_{m}[n-k]+\sigma_{q} \varepsilon_{q}[n],
$$

where $\varepsilon_{q}[n]$ is additive white Gaussian noise (AWGN) with zero-mean and unit variance, $\sigma_{q}$ denotes the actual noise standard deviation, which is assumed to be known, and $\beta_{m, q}[k]$ is the coefficient of the $q$-th output associated to the $k$-th shift of the $m$-th activation shape, $G_{m}(t)$. These activation shapes are modelled as samples from truncated Gaussian functions. More specifically, we define

$$
G_{m}(t)=\frac{1}{\sqrt{2 \pi \sigma_{m}^{2}}} e^{-t^{2} / 2 \sigma_{m}^{2}} \quad \text { for } \quad-t_{m} \leq t \leq t_{m}
$$

with $t_{m}$ a user-defined threshold (set up in practice so that $G_{m}\left( \pm t_{m}\right)$ is close to zero), and $\sigma_{m}^{2}$ a finite set of $M>R$ user-defined variances with $\sigma_{1}^{2}<\sigma_{2}^{2}<\ldots<\sigma_{M}^{2}$, and use discretized translated versions $G_{m}[n-k]=G_{m}\left((n-k) T_{s}-\right.$ $\left.t_{m}\right)$.

This model can be rewritten more compactly in matrix form by defining a set of matrices, $\mathbf{A}_{k}$ for $0 \leq k \leq N-1$, such that their $(n, m)$-th element is $\mathbf{A}_{k}(n, m)=G_{m}[n-$ $k-M]$ for $1 \leq n \leq N$ and $1 \leq m \leq M$ with $M=$ $\left\lfloor t_{\max } / T_{s}\right\rfloor$ and $t_{\max }=\max _{m}\left\{t_{m}\right\}=t_{M}$. Concatenating all these matrices we obtain an overcomplete global dictionary, $\mathbf{A}=\left[\mathbf{A}_{0}, \mathbf{A}_{1}, \ldots, \mathbf{A}_{N-1}\right]$, and (5) can be expressed as

$$
\mathbf{z}_{q}=\mathbf{A} \boldsymbol{\beta}_{q}+\sigma_{q} \varepsilon_{q}
$$

where $\boldsymbol{\beta}_{q}$ and $\varepsilon_{q}$ in (7) are $N M \times 1$ column vectors composed of $N$ subvectors of size $M$. More precisely,

$$
\begin{aligned}
\boldsymbol{\beta}_{q} & =\left[\boldsymbol{\beta}_{1, q}^{\top}, \boldsymbol{\beta}_{2, q}^{\top}, \ldots, \boldsymbol{\beta}_{N, q}^{\top}\right]^{\top}, \\
\boldsymbol{\beta}_{k, q} & =\left[\beta_{1, q}[k], \ldots, \beta_{M, q}[k]\right]^{\top}, 0 \leq k \leq N-1 \\
\varepsilon_{q} & =\left[\varepsilon_{1, q}^{\top}, \boldsymbol{\varepsilon}_{2, q}^{\top}, \ldots, \boldsymbol{\varepsilon}_{N, q}^{\top}\right]^{\top}, \\
\varepsilon_{k, q} & =\left[\varepsilon_{1, q}, \ldots, \varepsilon_{M, q}\right]^{\top}, 0 \leq k \leq N-1 .
\end{aligned}
$$

Note that this dictionary is not fitted to detect activation times being close to the signal boundaries $(k=0$ and $k=N-$ 1).However, this issue can be easily circumvented by adding zeros to the signal to process before $z[1]$ and after $z[N]$.

In order to obtain a sparse regressor, from which the information on the arrival times can be retrieved, we estimate $\boldsymbol{\beta}_{q}$ by means of LASSO [8]. Namely, $\hat{\boldsymbol{\beta}}_{q}^{L}(\lambda)$ is given by

$$
\hat{\boldsymbol{\beta}}_{q}^{L}(\lambda)=\underset{\boldsymbol{\beta}_{q} \in \mathbb{R}^{N M}}{\arg \min } \frac{1}{2 N}\left\|\mathbf{z}_{q}-\mathbf{A} \boldsymbol{\beta}_{q}\right\|_{2}^{2}+\lambda\left\|\boldsymbol{\beta}_{q}\right\|_{1},
$$

where $\left\|\boldsymbol{\beta}_{q}\right\|_{1}$ denotes the $\ell_{1}$ norm of $\boldsymbol{\beta}_{q}$ and $\lambda$ indicates the trade-off between sparsity and estimation precision. In this way, $\hat{\boldsymbol{\beta}}_{q}^{L}(\lambda)$ is much sparser than the solution of OLS or ridge regression estimators, which rely both on $\ell_{2}$ penalties [10].

However, in order to obtain an even sparser representation that takes into account the physiological restrictions imposed on the signals, we introduce an additional step after the computation of $\hat{\boldsymbol{\beta}}_{q}^{L}(\lambda)$. The samples associated to the arrival times of the spikes are estimated recursively as follows:

$$
\begin{gathered}
\hat{n}_{k, q}=\underset{n=1, \ldots, N}{\arg \max }\left\{\left\|\boldsymbol{\beta}_{n, q}\right\|_{1} \mathbb{I}\left(\eta_{q}<\left\|\boldsymbol{\beta}_{n, q}\right\|_{1}<\left\|\boldsymbol{\beta}_{\hat{n}_{k-1, q}}\right\|_{1}\right)\right\} \\
\text { s.t. }\left|\hat{n}_{k, q}-\hat{n}_{m, q}\right|>\Delta_{\text {min }}, m=1 \ldots k-1,
\end{gathered}
$$

where $\mathbb{I}(\cdot)$ is an indicator function (i.e. its value is one if the logical condition is fulfilled and zero otherwise), and $\eta_{q}$ and $\Delta_{\text {min }}$ are user-defined thresholds. The first one, $\eta_{q}$, is used to discard the $\boldsymbol{\beta}_{n, q}$ with a small $\ell_{1}$ norm, which contribute to improve the signal reconstruction but provide little information on the localization of the spikes. We have found out empirically that choosing $\eta_{q}=3 \sigma_{q}$ provides good results. The second one, $\Delta_{\min }$, accounts for the fact that consecutive pulses cannot overlap. Thus, in practice $\Delta_{\min }$ is chosen in such a way that $\Delta_{\min } / f_{s} \approx 100 \mathrm{~ms}$, which is a standard value for the refractory period.

Using this set of $P$ arrival times we may construct an $a c$ tivation sequence (also called spike train) composed of Kronecker deltas at the locations of the activations, ${ }^{3}$

$$
\pi_{q}[n]=\sum_{k=1}^{P} \delta\left[n-\hat{n}_{k, q}\right] .
$$

This sequence will be used for the spectral analysis, since it allows us to get rid of the effect of the unknown channels, $h_{r, q}(t)$, or the particular dictionary used, given by $G_{m}(t)$.

\subsection{Spectral Analysis}

The spectral analysis performed is based on applying an iterative deflation approach to the FFT of $\pi_{q}[n]$, extracting peaks with decreasing amplitudes up to a user defined threshold.

\footnotetext{
${ }^{3}$ Note that we have not estimated $R$ yet. Hence, we cannot separate the contribution of each foci to (10) as we did in the original model, given by (4).
} 


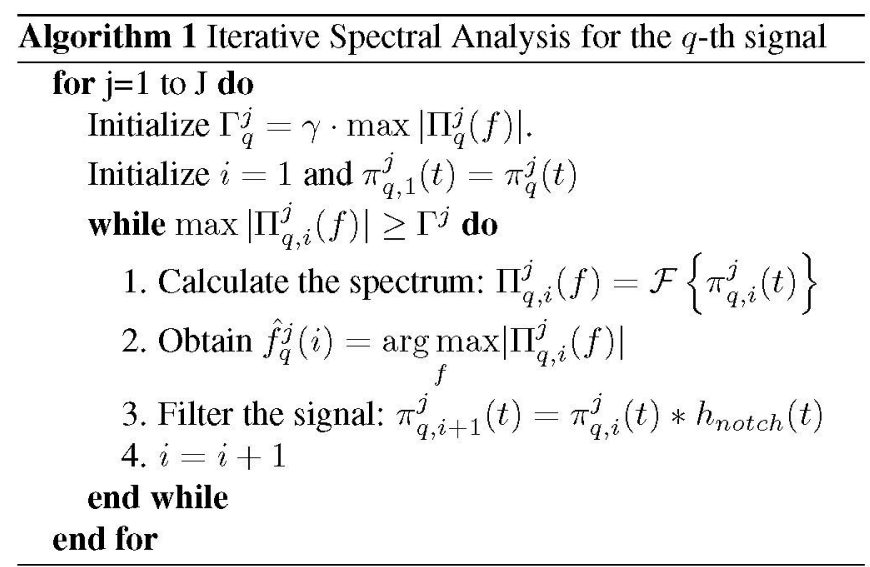

Hence, since we apply the spectral analysis to the inferred sparse activation sequence, we call our approach sparse spectral analysis (SSA). The number of peaks extracted (after the post-processing described in the following section) is an estimate of the number of existing foci and their locations provide us an estimate of their frequencies. ${ }^{4}$

Before describing the algorithm, let us remark that the analysis focuses on the frequency range that is physiologically interpretable. On the one hand, for sinus rhythm the heart rate can vary between 50 beats per minute (bpm) and $100 \mathrm{bpm}$, i.e. the range of valid frequencies is $0.5 \leq f_{r} \leq 2$ $\mathrm{Hz}$. On the other hand, when we analyze EGMs measured during AF, atrial cells fire typically at rates of $400-600 \mathrm{bpm}$ [1], leading to a useful frequency range $2 \leq f_{r} \leq 10 \mathrm{~Hz}$.

The first step in the SSA algorithm is segmenting $\pi_{q}[n]$ into $J$ windows containing $N_{s}=4 f_{s}$ samples (i.e. $\Lambda=4$ s) without overlap, as done in DFA (see Section 2). Then we apply Algorithm 1 to the FFT of each segment, $\Pi_{q}^{j}(f)=$ $\mathcal{F}\left\{\pi_{q}^{j}[n]\right\}$ with $1 \leq j \leq J$, after bandpass filtering. Algorithm 1 follows a deflation approach, searching iteratively for the highest peak of $\left|\Pi_{q}^{j}(f)\right|$ and adding it to the set of potential activation frequencies, $\hat{\mathbf{f}}_{q}^{j}$. After each iteration we apply a second-order IIR digital notch filter to the signal centered at the detected frequency with bandwith $B_{3 \mathrm{~dB}}=2 f_{\Lambda}=0.5 \mathrm{~Hz}$ to eliminate the detected peak before searching for a new one. The algorithm stops when the highest peak detected is below a threshold, $\Gamma_{q}^{j}=\gamma \max \left|\Pi_{q}^{j}(f)\right|$, being $\gamma$ a user defined parameter.

Figure 1 shows an example of the spectrum obtained iteratively for a single segment. The activation sequence, $\pi_{q}[n]$, has been syntheticaly generated using $R=3$ foci (with $f_{1}=$ $4 \mathrm{~Hz}, f_{2}=6 \mathrm{~Hz}$ and $f_{3}=7 \mathrm{~Hz}$ ) and random phases. The highest peak for the amplitude spectrum in the first iteration (shown in black) is $\hat{f}_{1} \approx 6.98 \mathrm{~Hz}$, which corresponds to $f_{3}$. Then we apply the notch filter centered around $\hat{f}_{1}$ to the signal, obtaining the amplitude spectrum shown in blue, and detecting $\hat{f}_{2} \approx 5.96 \mathrm{~Hz}$, which is close to $f_{2}$. After a sec-

\footnotetext{
${ }^{4}$ The location of the highest peak (i.e. the first one extracted) provides us with the dominant frequency.
}

ond notch filtering centered around $\hat{f}_{2}$, the third iteration (in green) detects $\hat{f}_{3} \approx 4 \mathrm{~Hz}$, which corresponds to $f_{1}$. After notch filtering again, iteration 4 (in red) detects $\hat{f}_{4} \approx 7.99 \mathrm{~Hz}$, which is the first harmonic of $\hat{f}_{3}$. Finally, after another notch filtering, all the peaks of the spectrum in the fifth iteration (in yellow) fall below the threshold $\Gamma=0.3 \times \max \left|\Pi_{q}(f)\right|$. Hence, the algorithm concludes after obtaining 4 potential frequencies: $\hat{\mathbf{f}}_{q}=[6.98,5.96,4,7.99] \mathrm{Hz}$.

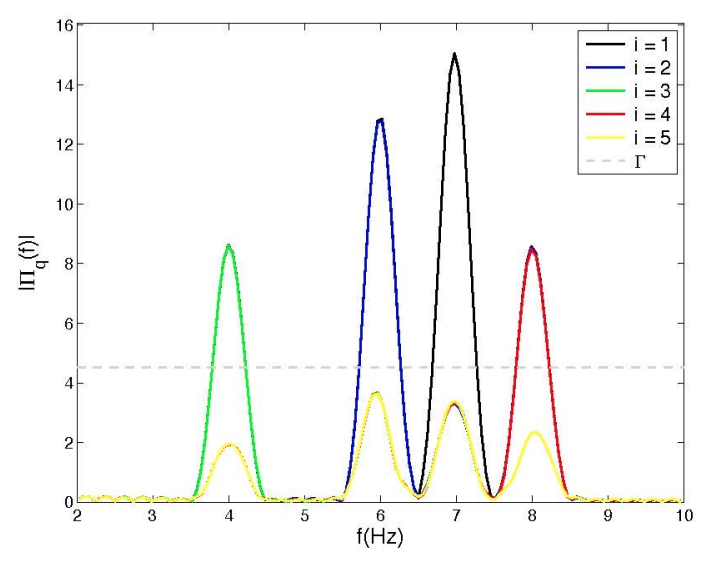

Fig. 1. Example of the SSA for a single segment of $\pi_{q}[n]$.

\subsection{Post-Processing: Discarding Harmonics}

The post-processing stage takes the set of potential activation frequencies detected inside each window, $\hat{\mathbf{f}}_{q}^{j}$, and determines whether they belong to different activation foci or not applying the following steps:

1. Elimination of repeated frequencies. Two frequencies, $f_{1}$ and $f_{2}$, correspond to the same focus if $\left|f_{1}-f_{2}\right| \leq$ $f_{\Lambda}$. If this happens, the one associated to the smallest peak is deleted.

2. Analysis of $2 / 3$ frequency relationships. Due to the frequency range used in the analysis, given a single frequency, $f_{0}$, in practice we can find at most two harmonics: $f_{1}=2 f_{0}$ and $f_{2}=3 f_{0}$. Thus, if we have detected the first and second harmonic of a given frequency, $f_{0}$, their relationship will be $f_{1}=\frac{2}{3} f_{2}$. Here we check this relationship, keeping only the frequency associated to a higher amplitude in the spectrum when we find it.

3. Discovery of harmonics and subharmonics. When two detected frequencies have a harmonic or subharmonic relationship, we only keep the one detected first.

4. Discovery of cross-modulation frequencies. We analyze whether each new element in $\hat{\mathbf{f}}_{q}$ is a crossmodulation product of two previously detected frequencies, i.e. whether $f_{3}= \pm m f_{1} \pm n f_{2}$ for any two integers $m$ and $n$. In this case $f_{3}$ will be deleted. 
With this analysis, we are able to estimate the number of activation foci present in our EGMs, $\hat{R}_{q}^{j}$, as well as their frequencies, $\hat{\mathbf{f}}_{q}^{j}$. Continuing with the example shown in Figure 1, the post-processing will find out that $\hat{f}_{4}$ is the first harmonic of $\hat{f}_{3}$, deleting it and obtaining a correct final estimation of $\hat{R}=3$ activation foci with frequencies $\hat{\mathbf{f}}_{q}=[6.98,5.95,4]$ $\mathrm{Hz}$, which are quite close to the true ones.

\section{RESULTS}

\subsection{Synthetic Data}

In this section we present an exhaustive analysis of the spectral analysis algorithm using synthetic data. In order to evaluate the performance of the spectral estimator, we generate synthetic activation sequences, $\pi_{q}[n]$, according to (10) with $f_{s}=977 \mathrm{~Hz}, N=16 f_{s}$ samples (i.e. 4 segments containing $N_{s}=4 f_{s}$ samples) and $Q=1$. We consider the possibility that some of the arrival times, $\hat{n}_{k, q}$, are undetected and model it using a fixed loss probability, $P_{\text {loss }}$. We test the algorithm for $R \in\{2,3,4\}$ foci, varying two parameters: $P_{\text {loss }}$ and the relative threshold parameter, $\gamma$. All the simulations have been performed averaging 1000 realizations with random phases. The activation frequencies, $f_{r}$, are randomly generated from a uniform distribution, i.e. $f_{r}(\mathrm{~Hz}) \sim \mathcal{U}([2,10])$, avoiding harmonic relationships, unlikely to occur in practice.

First of all, Figure 2 shows a receiver operating characteristic (ROC) type representation for $R=2$ and several values of $P_{\text {loss }}$ [11]. Here we have defined the false alarm probability as $P_{f a}=\operatorname{Pr}\{\hat{R}>R\}$ and the non-detection probability as $P_{n d}=\operatorname{Pr}\{\hat{R}<R\}{ }^{5}$. The performance of the SSA for detecting the number of foci is close to perfect for $P_{\text {loss }}=0$, gradually deteriorating as $P_{\text {loss }}$ increases, although for $P_{\text {loss }} \leq 0.1$ (the expected situation) the performance is still very good.

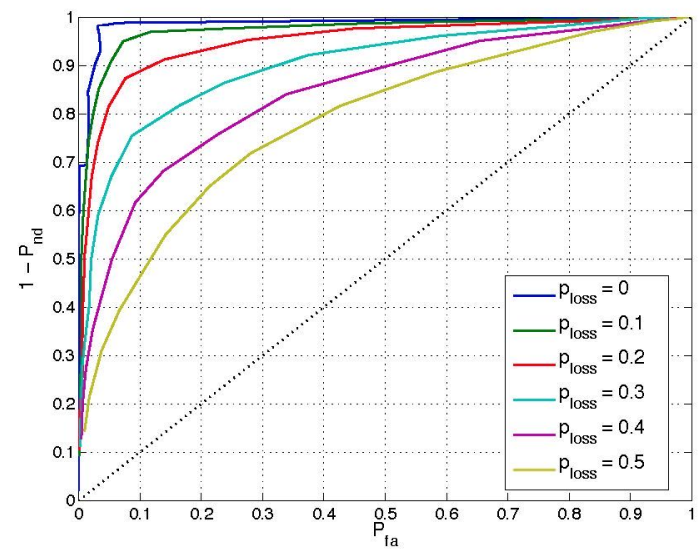

Fig. 2. ROC-type curve for $R=2$ foci.

\footnotetext{
${ }^{5}$ Strictly speaking, ROC curves can only be used to illustrate the performance of a binary classifier [11]. However, by defining $P_{f a}$ and $P_{n d}$ we can obtain useful information from a ROC-type curve.
}
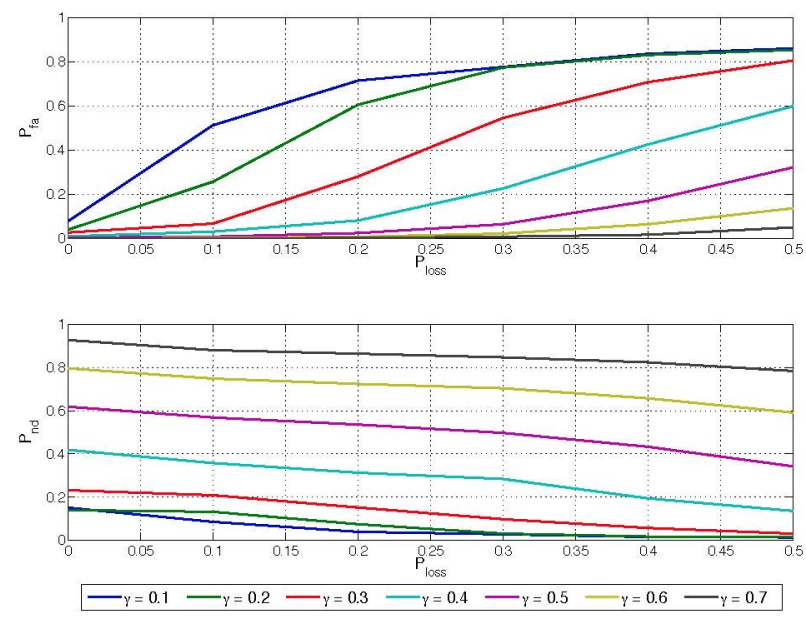

Fig. 3. $P_{f a}$ and $P_{n d}$ for $R=3$ as a function of $P_{l o s s}$.

\begin{tabular}{|c|c|c|c|c|c|c|}
\hline$P_{\text {loss }}$ & 0 & 0.1 & 0.2 & 0.3 & 0.4 & 0.5 \\
\hline$M S E\left(\times 10^{-3}\right)$ & 0.6 & 0.9 & 1.3 & 1.8 & 2.7 & 3.8 \\
\hline
\end{tabular}

Table 1. MSE for the frequency estimation with $R=4$ foci.

Although each point in Figure 2 corresponds to a certain value of $\gamma$, Figure 3 shows explicitly the behaviour of $P_{f a}$ and $P_{n d}$ (defined as above) for $R=3$ and several values of $\gamma$. Not surprisingly, when $\gamma$ decreases $P_{f a}$ increases and $P_{n d}$ decreases. Moreover, the higher the value of $P_{\text {loss }}$, the more likely is $\left|\Pi_{q}^{j}(f)\right|$ to contain spurious peaks, leading to larger values of $P_{f a}$ and smaller values of $P_{n d}$, indicating that the number of foci tends to be overestimated. However, for $P_{\text {loss }} \leq 0.1$ and the proper threshold choice, we obtain $P_{n d}<0.2$, which is quite satisfactory for this application.

Finally, in Table 1 we show the mean square error (MSE) for $R=4$, considering only the cases in which the correct number of foci and frequencies have been estimated. Although the MSE gets worse as $P_{\text {loss }}$ increases, for $P_{\text {loss }} \leq$ 0.1 the MSE is below $10^{-3}$, which is a very good result.

\subsection{Real Data: Sinus Rhythm and Atrial Fibrillation}

In this section we show two preliminary examples of the performance of the algorithm with real data. The signals used in this case correspond to bipolar intracardiac EGMs, obtained placing a set of electrodes (corresponding to a lasso catheter with $Q=10$ sensors and sampling frequency $f_{s}=977 \mathrm{~Hz}$ ) directly in contact with the heart muscle during surgery [2].

Figure 4 shows an example of the activations detected, both for a healthy heart under sinus rhythm and for an atrial fibrillation example. In the first case $\sigma_{q}=0.002$ for all channels and $\lambda=10^{-4}$, whereas for the second case we have used $\sigma_{q}=0.0005$ and $\lambda=10^{-6}$. The regularity of the sinus rhythm can be appreciated in Figure 4(a), with just a few 


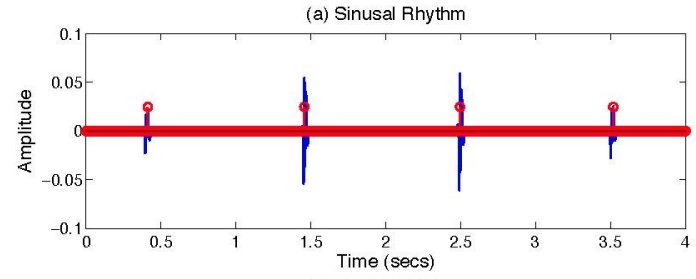

(b) Atrial Fibrillation

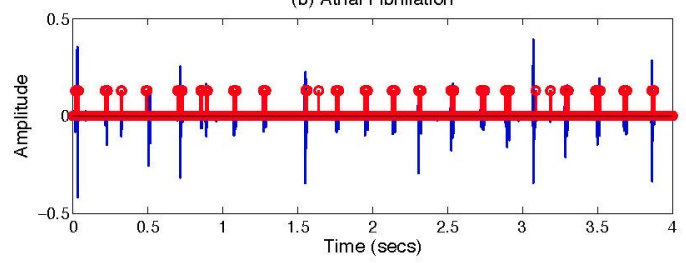

Fig. 4. Example of two EGMs (blue) and sparse activations (red).

periodic activations that are easily detected. In contrast, $\mathrm{AF}$ EGMs are much more challenging, with many peaks that are not easy to distinguish from noise. However, as it can be seen in Figure 4(b), the sparse inference algorithm described in Section 3.2 is able to detect all the major peaks.

Finally, we check the SSA using the first segment of the first channel with $\gamma=0.6$, the best threshold obtained from the synthetic data. For the sinus rhythm example, the SSA detects 3 potential frequencies, $\hat{\mathbf{f}}_{1}(\mathrm{~Hz})=[0.95,2.86,1.91]$, before post-processing. After removing the harmonics we finally attain $\hat{R}=1$ (as expected) and $\hat{f}_{1}=0.95 \mathrm{~Hz}$, which is the same value obtained for other segments and channels, as well as by DFA. Tests performed on other signal sets show similar results: a single activation with the same frequency obtained by DFA. For the AF example, the spectrum is shown in Figure 5. Applying the SSA we obtain $\hat{\mathbf{f}}_{q}(\mathrm{~Hz})=$ $[5.96,5.13,8.41,9.84,4.77]$ before post-processing. After removing the redundant frequencies we finally obtain $\hat{R}=4$ foci, with $\hat{\mathbf{f}}_{q}(\mathrm{~Hz})=[5.96,5.13,8.41,9.84]$. Although the estimation of $R$ seems to be robust, we have observed

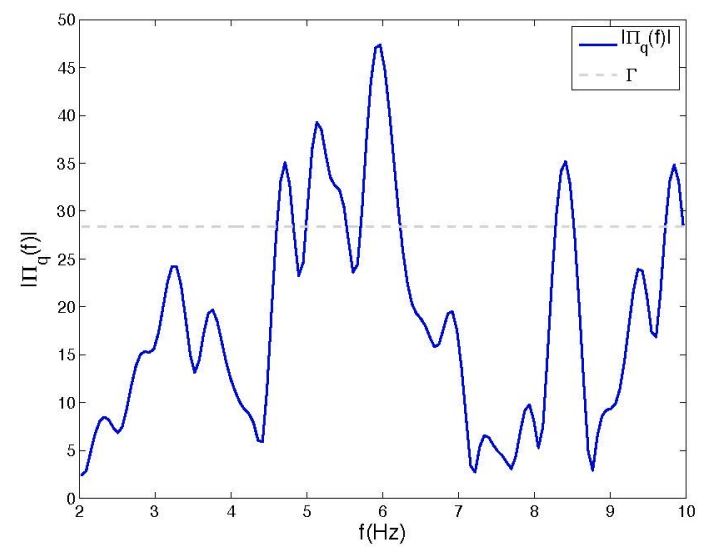

Fig. 5. Example of AF spectrum. important differences in the estimated frequencies for different segments and channels. For example, in the second segment of the first channel we obtain $\hat{R}=4$ foci and $\hat{\mathbf{f}}_{q}(\mathrm{~Hz})=[5.19,5.84,6.98,9.6]$. Note that the DFs obtained in these two examples are $5.96 \mathrm{~Hz}$ and $5.19 \mathrm{~Hz}$ respectively, which correspond to the highest peaks detected by the SSA.

\section{CONCLUSION}

In this paper we have proposed a novel methodology for characterizing atrial fibrillation electrograms. We hypothesize that the observed signals are the result of $R$ unobserved latent foci and perform a sparse spectral analysis for estimating $R$ and inferring the activation frequencies. The proposed approach has been tested both on synthetic and real data with good results, although more experiments are required to further validate the method. Other future lines include testing functions more tailored to the data for the sparse analysis (e.g. wavelets), introducing spectral analysis methods based on eigen-values or developing a multi-channel approach.

\section{REFERENCES}

[1] S. Nattel, "New ideas about atrial fibrillation 50 years on," Nature, vol. 415, pp. 219-226, Jan. 2002.

[2] P. Sanders et al., "Spectral analysis identifies sites of highfrequency activity maintaining atrial fibrillation in humans," Circulation, vol. 112, no. 6, pp. 789-797, August 2005.

[3] O. Barquero-Pérez et al., "Fundamental frequency and regularity of cardiac electrograms with Fourier organization analysis.," IEEE Trans. Biomedical Eng., vol. 57, no. 9, pp. 21682177, 2010.

[4] J. Ng, A. H. Kadish, and J. J. Goldberger, "Technical considerations for dominant frequency analysis," J. of Cardiovascular Electrophysiology, vol. 18, no. 7, pp. 757-764, July 2007.

[5] Gerald Fischer et al., "On computing dominant frequency from bipolar intracardiac electrograms," IEEE Trans. on Biomedical Engineering, vol. 54, no. 1, pp. 165-169, January 2007.

[6] L. T. Mainardi et al., "Linear and non-linear analysis of atrial signals and local activation period series during atrialfibrillation episodes," Medical and Biological Engineering and Computing, vol. 39, no. 2, pp. 249-254, 2001.

[7] M. P. Nguyen et al., "A new approach for automated location of active segments in intracardiac electrograms," in World Congress on Medical Physics and Biomedical Eng. 2009.

[8] R. Tibshirani, "Regression shrinkage and selection via the LASSO," Journal of the Royal Statistical Society, vol. 58, no. 1, pp. 267-288, 1996.

[9] T. Trigano et al., "On nonhomogeneous activity estimation in Gamma spectrometry using sparse signal representation," in IEEE Statistical Signal Proc. Wkshp., Jun. 2011, pp. 649-652.

[10] T. Hastie et al., The Elements of Statistical Learning: Data Mining, Inference, and Prediction, Springer, Feb. 2009.

[11] H. L. Van Trees, Detection, Estimation, and Modulation Theory. Part I, John Wiley \& Sons, New York, 2001. 